\title{
Impact of blood volume on the diffuse reflectance spectra of human skin in visible and NIR spectral ranges
}

E. Zherebtsov, A. Doronin, A. Bykov, A. Popov, I. Meglinski

E. Zherebtsov, A. Doronin, A. Bykov, A. Popov, I. Meglinski, "Impact of blood volume on the diffuse reflectance spectra of human skin in visible and NIR spectral ranges," Proc. SPIE 10412, Diffuse Optical Spectroscopy and Imaging VI, 104120H (28 July 2017); doi: 10.1117/12.2280634

SPIE. Event: European Conferences on Biomedical Optics, 2017, Munich, Germany 


\title{
Impact of Blood Volume on the Diffuse Reflectance Spectra of Human Skin in Visible and NIR Spectral Ranges
}

\author{
E. Zherebtsov"1, A. Doronin ${ }^{2}$, A. Bykov ${ }^{3}$, A. Popov ${ }^{3}$, I. Meglinski ${ }^{3}$ \\ ${ }^{I}$ Aston University, Optoelectronics and Biomedical Photonics Group, Aston Institute of Photonic Technologies, \\ Aston Triangle, Birmingham B47ET, UK \\ ${ }^{2}$ Yale University, Department of Computer Science, Computer Graphics Group, New Haven, CT, 06520, USA \\ ${ }^{3}$ University of Oulu, Opto-Electronics and Measurement Techniques Research Unit, Faculty of Information Technology and \\ Electrical Engineering, P.O. Box 4500, 90014 Oulu, Finland \\ *Correspondence: e.zherebtsov@aston.ac.uk
}

\begin{abstract}
With pulse wave periodic beating taken into account we assess the influence of blood volume and oxygen saturation changes on the measurements of diffuse reflectance spectra of human skin in the visible and NIR spectral range.

OCIS codes: (000.4430) Numerical approximation and analysis; (170.6510) Spectroscopy, tissue diagnostic
\end{abstract}

\section{Introduction}

At present, diffuse light reflectometry from different biological tissues in vivo and in vitro is widely employed as a measurement technique. This modality provides valuable quantitative and qualitative information about the abundance of components such as fractions of hemoglobin, bilirubin etc. The use of visible $(400-700 \mathrm{~nm})$ or NIR $(700-1400 \mathrm{~nm})$ spectral ranges allows conducting such measurements non-invasively and almost in real time. ${ }^{1,2}$ The reflectance is used in such diagnostic technologies in a form of the steady-state diffuse reflectance spectroscopy, ${ }^{3}$ diffusing-wave spectroscopy, diffuse correlation spectroscopy, fluorescence spectroscopy, ${ }^{4,5}$ pulse oximetry ${ }^{6}$ etc. The novel application of the different types of diffuse spectroscopy is for development of novel diagnostical wearable devices. The application of this measurement technique is promising due to the simplicity of realization of such devices and their compactness. ${ }^{7}$ In all cases of using optical measurements to assess parameters of a biological object in vivo and in situ, it is important to know the actual sampling volume (SV) of the biological tissue. In the general case of diffuse reflectance measurements, the sampling volume is the three-dimensional spatial region of a biological tissue with significant number of optical paths of detected photons. Technical parameters of the employed diagnostic system (probing geometry and wavelength of probing radiation), as well as parameters of the biological tissue, are major factors that influence the size and shape of the volume.

An alteration of the blood volume fraction, as well as oxygen saturation level (in case of measurements beyond isobestic points of $\mathrm{Hb} / \mathrm{HbO}_{2}$ pair) have impact on the geometrical parameters of the sampling volume. At the same time, the variations of blood and saturation in tissues are natural process for every living animal organism. Basically, the main causes of alterations are cardiac function, breathing as well as different types of vessel regulations. In addition, the pressure applied on optical diagnostic probes can be a significant factor affecting the local blood volume fraction. ${ }^{8}$

Thus, the aim of this work was to study the effect of sampling volume pulsation during diffuse reflectance measurements, arising from pulsation of the blood volume fraction as well as pulsation of blood saturation.

\section{Methods and Materials}

One of the possible ways to formalize calculation of the sampling volume is the assessment of the spatial region, which forms spatial depth sensitivity of the detector. The quantity is defined as the gradient of the optical density with respect to absorption coefficient $\mu_{a}$ at each pixel $\vec{r}$ in the medium ${ }^{9}$ :

$$
Q(\vec{r})=\frac{\partial}{\partial \mu_{a}(\vec{r})} \ln \left(\frac{I}{I_{0}}\right) .
$$

Where $\mu_{a}(\vec{r})$ is the absorption coefficient, $I_{0}$ is the intensity of probe radiation, $I$ is the intensity of detected radiation. The use of analytical mathematical models to figure out the parameter $Q$ for complex multilayer tissues (such as skin), is accompanied by a significant error in the calculations. The more precise approach involves numerical solving of the problem by the Monte Carlo method. ${ }^{10}$ The technique can provide a realistic distribution of light propagation in biological tissues. Monte Carlo simulation consists of a sequential random walk of photon packets between scattering events from the site of photon injection into the medium to the site where the photon leaves the medium.

Diffuse reflectance measurements can be implemented by different instruments with spectrometers with a diffraction grating and fiber-optical probes being the most common. In this study, the diffuse reflectance measurements of the 
QR400-7SR (Ocean Optics, Inc.) probe sampling volume were modelled and analyzed. The probe has 6 emitting fibers and one collecting fiber. Both types of the fibers are $400 \mu \mathrm{m}$ in diameter. Separation between the collecting and the emitting fibers is set as $480 \mu \mathrm{m}$.

In this study, a CUDA-based Monte-Carlo distributed computing platform ${ }^{11}$ was used for routine simulation of detector depth sensitivity (sampling volume) and skin reflectance spectra, as well as their variations associated with physiological changes in the human skin. The variations in the spatial distribution of blood, melanin, oxygen saturation of blood, hematocrit, water content within the skin, as well as the numerical aperture and angle of the detector positioning on the skin surface were taken into account. To assess sampling volume of the measurements by the probe, a seven-layer model of skin was used. The main parameters of the model are presented in Table 1.

Table 1. Parameters used for assessment of the absorption coefficients of the layers. ${ }^{12,13}$

\begin{tabular}{|l|c|c|c|c|c|}
\hline \multicolumn{1}{|c|}{ Layer } & Thickness, $\mu \mathrm{m}$ & $\begin{array}{c}\text { Blood volume } \\
\text { fraction } C_{\text {blood }} \%\end{array}$ & $\begin{array}{c}\text { Saturation, } \\
\%\end{array}$ & $\begin{array}{c}\text { Water, } \\
\%\end{array}$ & $\begin{array}{c}\text { Melanin, } \\
\%\end{array}$ \\
\hline Stratum corneum & 20 & 0 & 0 & 5 & 0 \\
\hline Living epidermis & 60 & 0 & 0 & 20 & 2 \\
\hline Papillary dermis & 250 & $5 \mathrm{~Kb}$ & Sat & 30 & 0 \\
\hline $\begin{array}{l}\text { Upper blood net } \\
\text { dermis }\end{array}$ & 330 & $20 \mathrm{~Kb}$ & Sat & 40 & 0 \\
\hline Reticular dermis & 1830 & $4 \mathrm{~Kb}$ & Sat & 50 & 0 \\
\hline $\begin{array}{l}\text { Deep blood net } \\
\text { dermis }\end{array}$ & 1910 & $10 \mathrm{~Kb}$ & Sat & 50 & 0 \\
\hline Subcutaneous fat & 8000 & $7 \mathrm{~Kb}$ & Sat & 60 & 0 \\
\hline
\end{tabular}

Blood pulsation was modelled by alteration of parameter $K b$ in the range of $0.85-1$. Saturation pulsation was modeled by the alteration of the parameter Sat in the range of $0.85-0.95$. Modulation of the blood volume fraction as well as blood saturation, were completed using the photoplethysmography function. The calculations were carried out for the following wavelengths: $434 \mathrm{~nm}, 450 \mathrm{~nm}, 480 \mathrm{~nm}, 500 \mathrm{~nm}, 545 \mathrm{~nm}, 560 \mathrm{~nm}, 660 \mathrm{~nm}$, and $800 \mathrm{~nm}$.

In the used model, the absorption coefficients of each layer take into account the concentration of blood $C_{\text {blood }}$ in various vascular beds, oxygen saturation $S$, water content $C_{H 2 O}$ and melanin fraction $C_{m e l} .^{12,13}$ To plot results of the modelling, the obtained values of the quantity $Q$ were summarized in a direction normal to plane of projection: $I_{x, z}=\sum_{y=1}^{N} Q_{x, y, z}$, where $Q_{x, y, z}$ is the modeled spatial depth sensitivity of the detector. After that the obtained 2D matrix was logarithmically scaled: $I_{i, j}^{I}=\log _{10}\left(I / I_{\max }\right)$.

\section{Result and Discussion}

The results of modelling of the sampling volume for the plane perpendicular to the line connecting the centers of the receiving and the transmitting fiber are presented in Fig. 1. It is seen that for light of the blue range (434 nm) the diagnostic depth does not exceed $250 \mu \mathrm{m}$, whereas for the red light it can exceeds $1.5 \mathrm{~mm}$. The mean calculated sampling volumes for different wavelengths of the probing radiation are presented in Tab. 2. The data were obtained taking into account the influence of all 6 emitting fibers in the probe. From Tab. 2, an obvious relationship can be deduced, where the sampling volume rises with the increasing wavelength. The exemptions of the rule are $480 \mathrm{~nm}$, $545 \mathrm{~nm}$ and $560 \mathrm{~nm}$ wavelength due to $Q$-bands of the strong absorption by of $\mathrm{Hb} / \mathrm{HbO}_{2}$. Also, due to the blood absorption, the sampling volume for $660 \mathrm{~nm}$ wavelength is bigger than one for $800 \mathrm{~nm}$.

The wavelengths used in the modelling can be categorised into 3 types: approximately isobestic points, $\mu_{a}^{H b}(\lambda) \approx \mu_{a}^{H b O 2}(\lambda)(450 \mathrm{~nm}, 500 \mathrm{~nm}, 545 \mathrm{~nm}, 800 \mathrm{~nm})$; points where oxyhemoglobin absorbs stronger than deoxyhemoglobin, $\mu_{a}^{H b}(\lambda)<\mu_{a}^{H b O 2}(\lambda)(480 \mathrm{~nm})$ and points where deoxyhemoglobin is a stronger absorber than oxyhemoglobin, $\mu_{a}^{H b}(\lambda)>\mu_{a}^{H b O 2}(\lambda)(434 \mathrm{~nm}, 560 \mathrm{~nm}, 660 \mathrm{~nm})$. Absolute values of the pulsatile calculated sampling volume for different wavelength during the pulse period are presented in Fig. $2 \mathrm{a}$ and their relative increment are presented in Fig. $2 \mathrm{~b}$. For the isobestic points, the influence of the blood oxygenation level on the value of the sampling volume is negligibly small. When $\mu_{a}^{H b}(\lambda)<\mu_{a}^{H b O 2}(\lambda)(480 \mathrm{~nm})$, a joint increase of both the blood volume fraction and the oxygenation leads to a significant decrease of the sampling volume. On the contrary, in Fig. $2 \mathrm{~b}$ it is clearly seen that for certain wavelengths of the probing radiation (434 nm and $660 \mathrm{~nm}$ ), where $\mu_{a}^{H b}(\lambda)>\mu_{a}^{H b O 2}(\lambda)$ and for the selected parameters of the pulse modelling, simultaneous increase of the blood volume fraction and saturation results in the increased sampling volume. Thus, in this conditions, decrease of light 
absorption due to increase of the oxygenation prevails over the absorption increase with the raise of blood content in the tissue during the pulse period. It is also possible that the influence of the increased blood volume fraction prevails over the oxygenation influence $(560 \mathrm{~nm})$. Thus, it appears reasonable to say that there is a set of (improbable) conditions, when the sampling volume remains constant during significant blood pulsation.

Table 2. Mean calculated sampling volume for the selected optical probe for different wavelengths of the probing light.

\begin{tabular}{|c|c|c|c|c|c|c|c|c|}
\hline$\lambda, \mathrm{nm}$ & 434 & 450 & 480 & 500 & 545 & 560 & 660 & 800 \\
\hline $\mathrm{SV}, \mathrm{mm}^{3}$ & 0.37 & 0.77 & 0.48 & 1.80 & 1.05 & 1.30 & 28.0 & 18.5 \\
\hline
\end{tabular}

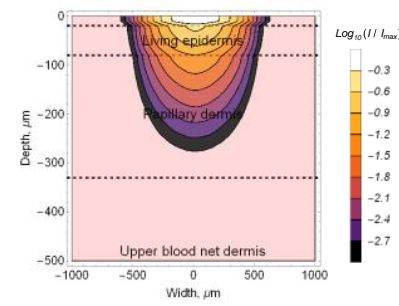

a) $\lambda=434 \mathrm{~nm}$

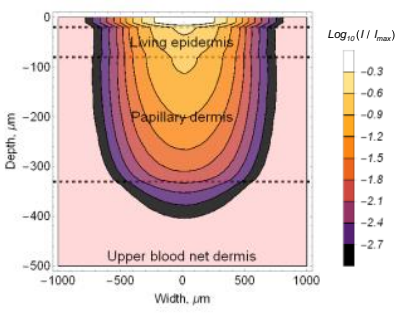

e) $\lambda=545 \mathrm{~nm}$

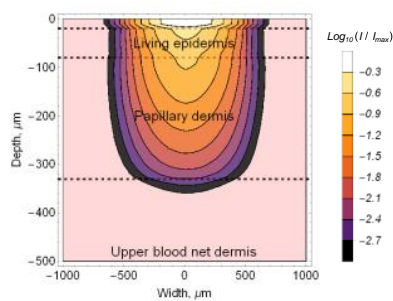

b) $\lambda=450 \mathrm{~nm}$

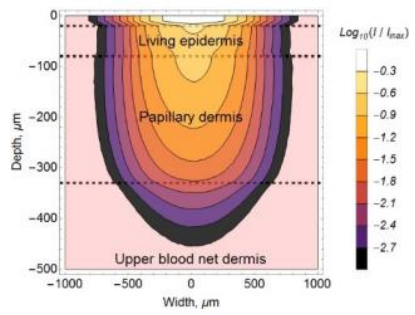

f) $\lambda=560 \mathrm{~nm}$

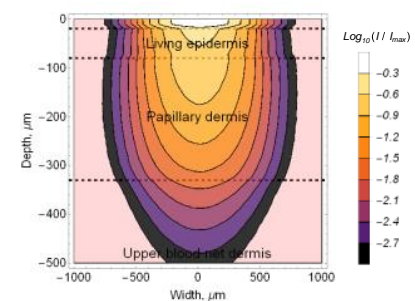

c) $\lambda=480 \mathrm{~nm}$

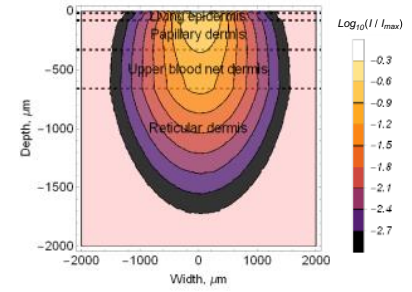

g) $\lambda=660 \mathrm{~nm}$

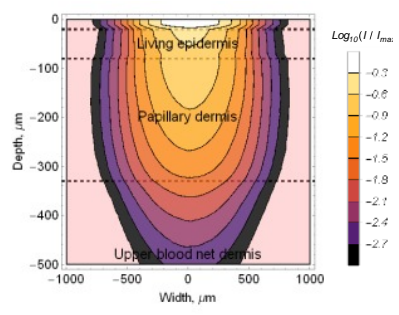

d) $\lambda=500 \mathrm{~nm}$

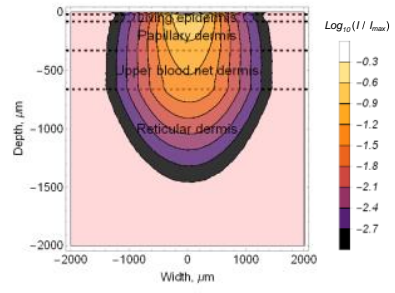

h) $\lambda=800 \mathrm{~nm}$

Figure 1. Calculated sampling volume of diffuse reflectance measurements for the modelled optical probe for several wavelengths of blue, yellow and green optical ranges. Wavelengths of the modeling are $434 \mathrm{~nm}(\mathrm{a}), 450 \mathrm{~nm}(\mathrm{~b}), 480 \mathrm{~nm}(\mathrm{c}), 500 \mathrm{~nm}(\mathrm{~d}), 545 \mathrm{~nm}(\mathrm{e}), 560 \mathrm{~nm}(\mathrm{f}), 660 \mathrm{~nm}(\mathrm{~g})$ and NIR $-800 \mathrm{~nm}(\mathrm{~h})$.

Furthermore, the results were presented in the form of animated graphs of sampling volume changes for scaling of the parameters of the main human skin layers related to the experimental results.

\section{Conclusion}

We consider changes in the volume of blood and oxygen saturation caused by a pulse wave and their influence on the diffuse reflectance spectra in the visible/NIR spectral range. A CUDA-based Monte Carlo model was used for routine simulations of the detector depth sensitivity (sampling volume) and skin spectra, and their variations associated with physiological changes in the human skin. The results are presented in the form of animated graphs of sampling volume changes for scaling of the parameters of the main human skin layers. Related to the experimental results, they are of particular interest for pulse oximetry, photoplethysmography, Doppler flowmetry, reflectance spectroscopy etc.

\section{Acknowledgments}

Authors acknowledge the support of the Academy of Finland (grants 296408 and 290596). EvZ acknowledges for personal support the funding from the European Union's Horizon 2020 research and innovation programme under grant agreement No 703145.

\section{References}

[1] A. V Dunaev et al., "Substantiation of medical and technical requirements for noninvasive spectrophotometric diagnostic devices.," J. Biomed. Opt. 18(10), 107009 (2013).

[2] A. V Dunaev et al., "Individual variability analysis of fluorescence parameters measured in skin with different levels of nutritive blood flow," Med Eng Phys 37(6), 574-583 (2015).

[3] V. O. Korhonen et al., "Light Propagation in NIR Spectroscopy of the Human Brain," IEEE J. Sel. Top. Quantum Electron. 20(2), 289-298 (2014).

[4] V. V Dremin et al., "The blood perfusion and NADH/FAD content combined analysis in patients with diabetes foot," Proc. SPIE 9698, 969810-969817 (2016).

[5] V. V Dremin et al., "The development of attenuation compensation models of fluorescence spectroscopy signals," Proc. SPIE 9917, 99170Y (2016).

[6] A. Doronin, I. Fine, and I. Meglinski, "Assessment of the calibration curve for transmittance pulse-oximetry," Laser Phys. 21(11), 1972-1977 (2011). 
[7] E. Zherebtsov et al., "Optical non-invasive diagnostics of microcirculatory-tissue systems of the human body: questions of metrological and instrumentation provision," J. Biomed. Photonics Eng. 2(4), 40305 (2016).

[8] E. A. Zherebtsov et al., "The influence of local pressure on evaluation parameters of skin blood perfusion and fluorescence," Proc. SPIE 10336, 1033608 (2017).

[9] I. V Meglinsky and S. J. Matcher, "Modelling the sampling volume for skin blood oxygenation measurements," Med. Biol. Eng. Comput. 39(1), 44-50 (2001).

[10] A. V. Bykov et al., "Monte carlo simulation of signals from model biological tissues measured by an optical coherence tomograph and an optical coherence Doppler tomograph," Opt. Spectrosc. 101(1), 33-39 (2006).

[11] A. Doronin and I. Meglinski, "Online object oriented Monte Carlo computational tool for the needs of biomedical optics," Biomed. Opt. Express 2(9), 2461 (2011).

[12] G. I. Petrov et al., "Human tissue color as viewed in high dynamic range optical spectral transmission measurements.," Biomed. Opt. Express 3(9), 2154-2161 (2012).

[13] I. Meglinski et al., [Computational Biophysics of the Skin] "Dermal Component - Based Optical Modeling of Skin Translucency: Impact on Skin Color," Pan Stanford Publishing, 25-61 (2014). 